\title{
The Impact of eHealth on the Quality and Safety of Healthcare
}

\author{
Azeem Majeed*, Ashly Black, Josip Car, Chantelle Anandan, Kathrin Cresswell, \\ Brian McKinstry, Claudia Pagliari, Rob Procter, and Aziz Sheikh \\ * Imperial College London, London, UK \\ University of Edinburgh, Edinburgh, UK
}

\begin{abstract}
There is considerable interest in using information technology (IT) to enhance the quality and safety of healthcare. We undertook a systematic literature review to assess the impact of eHealth applications on the quality and safety of healthcare. We retrieved 46,349 potentially relevant publications, from which we selected 67 relevant systematic reviews for inclusion. The literature was found to be poorly collated and of variable quality in its methodology, reporting and utility. We categorised eHealth applications into three main areas: i). storing, managing and transmission of data; ii). supporting clinical decisionmaking; and iii). facilitating care from a distance. We found that relative to the potential benefits noted within the literature, little empirical evidence exists in support of these applications. Of the few studies revealing the clearest evidence of benefits, many are from academic clinical centres where developers of new applications have also been directly associated with their evaluation. It is therefore unclear how effective these applications would be if deployed outside the environment in which they were developed. Our review of the impact of eHealth applications on quality and safety of healthcare demonstrated a vast gap between the postulated and empirically demonstrated benefits. In addition, there is a lack of robust research on risks and costs. Consequently, the costeffectiveness of these interventions has yet to be demonstrated.
\end{abstract}

